In other conditions associated with placental oedema-such as the fetofetal transfusion syndrome, and hydrops fetalis due to rhesus isoimmunisation or congenital syphilis-low levels of the maternally synthesised immunoglobulin IgG have been found in the cord bloods of the infants. ${ }^{78}$ These findings suggest that placental transfer of $\mathrm{IgG}$ is also reduced.

We, like Chiang and Wei, ${ }^{7}$ found that the cord IgG levels were below the normal range for a white population (72-175 IU/ml). As studies have shown that mothers in tropical countries, and therefore their babies, tend to have higher levels of IgG, it is likely that the levels are even further outside the normal range for a Thai population. Unfortunately no data are available, but studies on unaffected infants and their mothers from Taiwan ${ }^{7}$ support this suggestion.

The whole complex pathophysiology of oedema in the fetus and newborn infant needs further study. Alpha-thalassaemic hydrops fetalis is an example of the most extreme form of intrauterine oedema and provides a unique model for investigation of the underlying mechanisms of oedema formation. Now that rhesus isoimmunisation can be prevented (or its effects altered in utero), there is no other condition in which hydrops fetalis can be predicted and thus be accessible to study from early pregnancy.

We thank Dr S Silpisornkosol for allowing us to study patients under her care, Dr T Seesuwan for collecting the blood samples, and Dr David Baum for help and encouragement.

Dr Elizabeth Bryan was financially supported by H J Heinz Limited.

\section{References}

1 Thumasathit B, Nondasuta A, Silpisornkosol S, Lousuebsakul B, Unchalipongse $\mathbf{P}$, Mangkornkanok $\mathbf{M}$. Hydrops fetalis associated with Bart's hemoglobin in northern Thailand. J Pediatr 1968; 73: 132-8.

2 Wasi P, Na-Nakorn S, Pootrakul S-N. The $\alpha$ thalassaemias. Clin Haematol 1974; 3: 383-410.

3 Phibbs R H, Johnson P, Tooley W H. Cardiorespiratory status of erythroblastotic newborn infants. II. Blood volume, hematocrit, and serum albumin concentration in relation to hydrops fetalis. Pediatrics 1974; 53: 13-23.

4 Gray G R, Towell M E, Wright V J, Hardwick D F. Thalassemic hydrops fetalis in two Chinese-Canadian families. Can Med Assoc J 1972; 107: 1186-90.

5 Baum J D, Harris D. Colloid osmotic pressure in erythroblastosis fetalis. $\mathrm{Br}$ Med J 1972; i: 601-3.

6 Barnes S E, Bryan E M, Harris D, Baum J D. Oedema in the newborn. In: Molecular aspects of medicine. Vol. 1, part 3. Oxford: Pergamon, 1977.

7 Chiang W T, Wei P Y. Immunoglobulins in hydrops fetalis. Am J Obstet Gynecol 1972; 114: 816-8.

8 Bryan E M. Serum immunoglobulins in twin pregnancy with particular reference to the fetofetal transfusion syndrome. MD thesis, University of London 1976.

Correspondence to Dr Elizabeth M Bryan, Department of Paediatrics and Neonatal Medicine, Hammersmith Hospital, Du Cane Road, London W12 0HS.

Received 29 July 1980

\title{
Factors influencing mortality and morbidity after clinically apparent intraventricular haemorrhage
}

\author{
RICHARD O ROBINSON AND NIRMALA S DESAI
}

Department of Pediatrics and Neurology, University of Kentucky

SUMMARY Twenty newborn infants with clinically apparent intraventricular haemorrhage were studied in order to determine the factors associated with mortality and morbidity. Ten survived, 4 without handicap and 2 with only moderate handicap. Maturity and not size of haemorrhage appeared to be the main factor affecting mortality and morbidity at 1 year. Coma longer than 24 hours after intraventricular haemorrhage distinguished survivors with handicap from those without and may be a useful prognostic sign.

Intraventricular haemorrhage (IVH) is a common event in preterm infants. ${ }^{1-3}$ In about half the cases it may be occult, detectable only by computerised tomography (CT) scans or ultrasound. When it is clinically evident, it is associated with a high mortality and, in the event of survival, with a high morbidity; ${ }^{4} 5$ survival may be complicated by hydrocephalus too. Changes in methods of life support now lead to greater numbers of survivors of IVH, many of whom are surviving with handicap. We have examined our experience in order to determine which factors influence mortality and morbidity from IVH.

\section{Methods}

Babies born between 1 January 1977 and 1 January 
1978 and cared for in the neonatal intensive care unit were identified as having IVH if (1) CT scan showed high-density material in lateral ventricles, (2) if necropsy demonstrated blood in the ventricles, or (3) if progressive hydrocephalus associated with CSF heavily stained with blood breakdown products developed. A suspicious clinical picture was not in itself sufficient even if it was accompanied by bloody CSF. A CT scan was performed only if the diagnosis of IVH was suspected on clinical grounds.

Arterial blood gas estimations and arterial pressure measurements were made by residents and nursing staff as often as the clinical state of the baby indicated, and, in the case of arterial pressure measurements, not less than every 2 hours. An attempt was made in each case to determine when IVH occurred. This was assumed when a sudden, otherwise inexplicable cardiovascular collapse and neurological deterioration took place in association with a fall in haematocrit and a rise in anterior fontanelle pressure, although clear evidence for IVH as defined above may not have been present until some time later. Although IVH was suspected at the time in such circumstances, efforts were made to restore acid-base balance and oxygenation, and to reverse hypotension. Death occurred only after further deterioration despite resuscitation efforts and not as the result of withdrawal of life support facilities. All but one of the survivors were examined by one or both of us, between 1 and 2 years' postnatal age, using standard clinical neurological and developmental assessment techniques (the exception being a child who could not be traced as his family had moved). The classification severely handicapped was used if learnt behaviour had been acquired at less than half the normal rate, or if spasticity or dystonia had prevented the acquisition of motor skills. Moderate handicap implied development at a rate 50 to $70 \%$ of the normal rate in the presence of motor, intellectual, or visual dysfunction. For purposes of comparison with the largest current series in which prospective CT scanning of low birthweight infants was undertaken, ${ }^{2}$ the size of haemorrhage was graded 1,2 , or 3 depending on whether <one-quarter, one-quarter to one-half, or $>$ one-half of the volume of the lateral ventricles was occupied by blood of sufficient density to be detected by the CT scanner.

\section{Results}

Mortality. Our experience of 20 children was analysed. Ten of them died and in 7 the death was directly attributable to IVH. In the 3 in whom it was not, 2 succumbed with meningitis and the third with
Table Details for the 17 babies with intraventricular haemorrhage

\begin{tabular}{lll}
\hline & $\begin{array}{l}\text { Survived } \\
(n=10)\end{array}$ & $\begin{array}{l}\text { Died } \\
(n=7)\end{array}$ \\
\hline $\begin{array}{l}\text { Gestational age (weeks) } \\
\text { Birthweight ( }(\mathrm{g})\end{array}$ & $33 \cdot 5 \pm 3 \cdot 1$ & $29.4 \pm 2 \cdot 1^{*}$ \\
$\begin{array}{l}\text { Intraventricular haemorrhage } \\
\text { Grade 1 }\end{array}$ & $1832 \pm 537$ & $1254 \pm 241^{* *}$ \\
$\begin{array}{l}\text { Grade 2 } \\
\text { Grade 3 }\end{array}$ & 1 & 0 \\
Subependymal haemorrhage & 3 & 2 \\
$\left.\begin{array}{l}* \mathbf{P}=0.0097 \\
* * \mathbf{P}=0.022\end{array}\right\}$ (Mann-Whitney U test). & 3 \\
\end{tabular}

Two babies who survived and one who died are excluded because IVH was inferred only from the development of posthaemorrhagic hydrocephalus.

an acute pulmonary haemorrhage. These 3 children have been excluded from subsequent analyses.

The main factor determining survival was gestational age. Babies who died were significantly less mature than babies who survived (Table). The two groups were separated to a lesser degree by the difference in their birthweights which presumably also reflected a difference in gestational age. Thus, although no baby $<30$ weeks' gestation with IVH survived, there were 4 survivors $<1500 \mathrm{~g}$ birthweight.

While size of haemorrhage was important it did not appear to dominate immediate outcome. In 3 babies the first CT scan after haemorrhage showed hydrocephalus only. Two of these babies and all babies with grade 1 haemorrhage survived. All but one of the babies who died had grade 2 or 3 IVH, the exception being a baby with a large subependymal haemorrhage affecting the head of the caudate nucleus and a portion of the white matter lateral to the caudate. However, 3 of the 6 babies with grade 3 haemorrhage survived. The degree of haemorrhage more acurately reflected the size and degree of maturity of the baby. All babies with grade 1 haemorrhage were more than $1800 \mathrm{~g}$ and more than 33 weeks' gestational age. By contrast all 10 babies lighter and less mature than this had grades 2 or 3 haemorrhage, but 4 of them survived.

It was possible to limit the timing of IVH in 6 survivors and 6 fatalities to one 24-hour period. Before IVH occurred the maximal arterial pressure in survivors was higher than in babies who subsequently died $(77 \cdot 3 \pm 12 \cdot 8$ versus $59 \cdot 0 \pm 11 \cdot 3$ $\mathrm{mmHg}, \quad P=0.037$ ). There was no significant difference between survivors and fatalities in extremes of blood gases, acid-base status, rectal temperature, serum sodium, or CSF glucose before or after IVH. Similarly, there was no difference between survivors and fatalities in amount or rate of alkali administration either before or after IVH. 
Morbidity. Four of the survivors are not handicapped, and 2 are handicapped to some extent. Five of the 10 survivors developed hydrocephalus and required shunting. Three of these infants required subsequent revision of the shunt. Three features were present in babies who survived with handicap, but were absent in babies who survived without handicap.

Although there seemed to be no difference with respect to the severity of collapse at the time of haemorrhage, speed of neurological recovery after resuscitation had restored the arterial pressure and acid base status was informative. All but one of the babies who survived with handicap remained hypotonic and unresponsive for more than 24 hours, the exception being a baby who recovered quickly but who went on to develop meningitis associated with shunt complications. By contrast, babies who survived without handicap were, without exception, responsive within 24 hours, although they were not always neurologically normal, some displaying either hypertonicity or spontaneous clonus.

A second difference emerged with respect to serum sodium. Those with handicaps had lower serum sodium concentrations at one stage of the illness than those surviving intact $(125.4 \pm 4.6$ versus $134.5 \pm$ $3.1 \mathrm{mmol} / 1, P=0.027)$. Maturity in survivors also affected outcome. Those handicapped were significantly more mature than those who survived intact (mean gestational age $35 \cdot 4 \pm 3 \cdot 1$ versus $31 \cdot 25 \pm 1 \cdot 9$ weeks, $P=0 \cdot 05$ ). The grade of haemorrhage bore no relation to the degree of morbidity in the survivors. Two of the 3 survivors with grade 3 haemorrhage survived without handicap although each had been born weighing $<1500 \mathrm{~g}$. Conversely, 3 of the 5 handicapped survivors had only grade 1 haemorrhages.

\section{Discussion}

Relatively minor degrees of IVH may go undetected unless all infants are subjected to routine CT scans. Ethical questions concerning resuscitation of babies with subclinical IVH do not by definition arise (although the prognosis of such infants is a matter of great interest). Consequently, this study was confined to clinically apparent IVH. Vigorous resuscitation of all such babies will increase the number of handicapped children. Is it possible to identify features close to the time of haemorrhage which will predict subsequent handicap? Ahmann et al. ${ }^{2}$ found that size of haemorrhage was related to immediate mortality and also to the development of posthaemorrhagic hydrocephalus. They have yet to report the development of their survivors. Krishnamoorthy et al., 5 perhaps not surprisingly, found that the most severe handicaps were associated with intraparenchymal haemorrhage. Grades 1 and 2 haemorrhage however, were not differentiated with respect to outcome. Given adequate and timely blood replacement there is no $a$ priori reason why the volume of blood in the ventricles should affect mortality unless other factors-such as a direct effect on respiratory or cardiovascular centres in the floor of the 4th ventricle - are invoked. If the findings of this study are substantiated however, it should not be assumed that grade 3 haemorrhage is necessarily associated with a poor outcome. Our findings suggest that size of haemorrhage should be considered together with gestational age. Although the size of haemorrhage is clearly important in that each baby who died had grade 2 or 3 haemorrhage, it was maturity that dominated outcome in this study. All babies who survived were of at least 30 weeks' gestational age, whereas all but 2 of the babies who died were less mature.

Similarly size of haemorrhage did not appear to affect morbidity in babies of more than 30 weeks' gestation. Maturity was again the dominating factor in that handicap among survivors was more common in more-mature babies in this group. One might speculate either that the more-mature infant successfully withstood greater cerebral damage or that the factors required to provoke intraventricular haemorrhage were more extreme in more-mature infants. Perhaps because of limited numbers, neither speculation was borne out by analyses of clinical observations before and after IVH and further work is required to clarify this relationship.

The second factor separating survivors with handicap from those without was a relative hyponatraemia which may represent inappropriate antidiuretic hormone secretion. This may occur either because an intracerebral event in some way unspecified stimulates the hypothalamic-hypophyseal axis or because immobility by impairing venous return reduces left atrial pressure. The degree of hyponatraemia was itself unlikely to impair brain function and the severity of the neurological events may influence either of these mechanisms.

Deonna et al. ${ }^{6}$ reported their experience of 11 survivors of IVH and concluded that severe retardation was associated with an episode of acute neurological deterioration. They suggested that in this situation vigorous resuscitation was not indicated. It should be noted however, that they were dealing with a subgroup of survivors in that the criteria for inclusion in their study was the development of posthaemorrhagic hydrocephalus. All but 2 of our survivors had severe neurological deterioration associated with cardiovascular collapse at the time of 
haemorrhage. Continuing coma for a period of 24 hours after adequate resuscitation was a grave prognostic sign and in this series clearly separates those surviving with handicap from those surviving intact. The cause of the coma is unclear. A study of cortical blood flow during this period would be of considerable interest since blood entering the subarachnoid space by the foramina of the 4th ventricle may cause arterial spasm and a secondary depression of cortical function. A report by Cooke et al. ${ }^{7}$ on apparent cerebral blood flow in the newborn, mentions observations on 2 very low birthweight babies with 'massive' IVH. In neither infant was apparent cerebral blood flow detectable after the onset of clinical signs suggesting IVH, but details of the neurological state were not given. It is possible that a closer study of babies after IVH may provide a basis for more rational treatment designed, not only to improve survival, but also to reduce morbidity.

\section{References}

1 Papile L-A, Burstein J, Burstein R, Koffler H. Incidence and evolution of subependymal and intraventricular hemorrhage: a study of infants with birth weights less than 1500 g. J Pediatr 1978; 92 : 529-34.

2 Ahmann P A, Lazzara A, Dykes F D, Brann A W, Schwartz J F. Intraventricular hemorrhage in the highrisk preterm infant: incidence and outcome. Ann Neurol 1980; 7: 118-24.

3 Volpe J. Intracranial hemorrhage in the newborn: current understanding and dilemmas. Neurology 1979; 29: 632-5.

4 Amiel C. Hémorrages cérébrales intraventriculares chez le prématuré. 2 partie. Les elements du diagnostic clinique. Biol Neonatorum 1965; 75: 57-75.

5 Krishnamoorthy K S, Shannon D C, DeLong G R, Todres I D, Davis $K R$. Neurologic sequelae in the survivors of neonatal intraventricular hemorrhage. Pediatrics 1979; 64: 233-7.

6 Deonna T, Payot M, Probst A, Prod'hom L S. Neonatal intracranial hemorrhage in premature infants. Pediatrics 1975; 56: 1056-64.

7 Cooke R W I, Rolfe P, Howat P. Apparent cerebral blood flow in newborns with respiratory disease. Dev Med Child Neurol 1979; 21: 154-60.

Correspondence to Dr R O Robinson, Newcomen Centre, Guy's Hospital, St Thomas Street, London SE1 9RT.

Received 9 July 1980

\section{Trimeprazine and respiratory depression}

\author{
NICHOLAS P MANN \\ City Hospital, Nottingham
}

SUMMARY Respiratory depression is described in two siblings after oral administration of less than the maximum recommended dose of trimeprazine tartrate.

Trimeprazine tartrate is commonly used in paediatric practice for premedication, sedation, and relief of pruritis. ${ }^{1}$ Acute poisoning may be associated with central nervous system depression and hypothermia. ${ }^{2}$ Extrapyramidal symptoms, elation or depression of mood and, rarely, agranulocytosis may follow recommended dosage. Two cases of severe respiratory depression in siblings are described after normal dosage of trimeprazine tartrate.

\section{Case reports}

Case 1. A 3-month-old, $6 \cdot 3-\mathrm{kg}$ boy, who had no history of respiratory illness, was admitted to hospital for repair of bilateral cleft lip and palate.
This was carried out successfully and there were no postoperative problems. Nasal packs had been removed and there was no evidence of any airway obstruction. Forty-eight hours postoperatively he was given an oral dose of $15 \mathrm{mg}$ trimeprazine tartrate $(2.4 \mathrm{mg} / \mathrm{kg})$ for night sedation. He became drowsy after administration of the drug and had a respiratory arrest $5 \frac{1}{2}$ hours later, requiring resuscitation using facial oxygen and skin stimulation for 3 to 4 minutes. A further respiratory arrest occurred 9 hours after the first, again responding to similar measures. Chest $x$-ray films showed no abnormality and no reason for the respiratory arrest was found. He made a full recovery without neurological or respiratory sequelae.

Case 2. The 3-year-old sister of Case 1, and weighing $12 \mathrm{~kg}$, had a minor surgical procedure for repair of a small fistula of the palate. She had had two operations previously for repair of her cleft lip and palate without any postoperative problems. $35 \mathrm{mg}(2 \cdot 9$ 